\title{
Electron Transfer Pathways and Dynamics in Drosophila Cryptochrome - the Role of Protein Electrostatics
}

\author{
Martin Richter ${ }^{1}$, and Benjamin P. Fingerhut ${ }^{1, *}$ \\ ${ }^{1}$ Max-Born-Institut für Nichtlineare Optik und Kurzzeitspektroskopie, Max-Born-Str. 2a, D-12489 \\ Berlin, Germany
}

\begin{abstract}
Dissipative quantum dynamics simulations reveal a branching of charge separation dynamics in Drosophila Cryptochrome due to subtle balanced energetics within the enzyme. In silico mutations of charged amino acids provide control over charge transfer directionality.
\end{abstract}

\section{Photoreception mechanism of Drosophila cryptochrome}

Cryptochromes are highly conserved flavoproteins consisting of an N-terminal photolyase homology region (PHR) that binds a flavin adenine dinucleotide (FAD) cofactor, and a C-terminal $\alpha$-helical domain with a variable C-terminal tail (CTT) that shows high diversity in sequence among organisms (Fig. 1a). Drosophila cryptochromes (dCRY) function as blue-light photoreceptors by synchronizing the circadian clock to the external stimuli of incident sunlight via conformational changes located in the CTT.

The microscopic details of the photoreception mechanism leading to CTT conformational changes upon light absorption of the FAD cofactor are unknown. It is generally assumed that photoreduction of FAD proceeds via a highly conserved triad of tryptophanes (dCRY Trp-triad: W420-W397-W342, Fig. 1b) where W420 acts as primary electron donor upon photoexcitation, in analogy to tryptophan-triad dependent photoactivation in photolyases. Such Trp-triad functionality has been recently questioned due to observations that dCRY promotes enzymatic activity either in the presence of $\mathrm{W} \rightarrow$ F mutations that abolish in vitro Trp-triad photoreduction [1].

Focussing on the elusive microscopic events initiating signal transduction upon photoexcitation of fully oxidized flavin $\mathrm{FAD}^{\mathrm{Ox}}$ as plausible resting state [2], state-of-the-art simulations are presented that consider charge separation via the Trp-triad and further take into account intra-FAD charge transfer involving the isoalloxazine (ISO) and adenine (ADE) moieties of the FAD co-factor, as well as charge transfer states involving spatially close W314 and W422 residues (cf. Fig. 1b), thus avoiding pre-bias to specific W-mediated charge separation pathways. Dissipative quantum dynamics simulations are conducted with microscopically derived model Hamiltonians accounting for dCRY thermal fluctuation dynamics. Selected in silico point mutations provide a microscopic basis for rationalizing

* Corresponding author: fingerhut@mbi-berlin.de 
electron transfer directionality and demonstrate a high degree of electrostatic control realized by discrete charged amino acid residues.
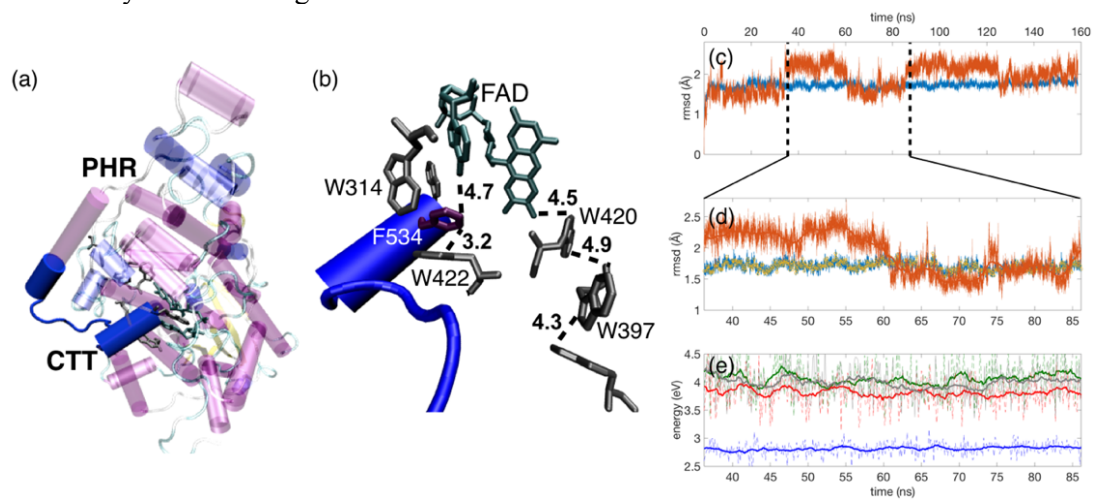

Fig. 1. (a) Structure of dCRY with helical C-terminal tail (CTT) highlighted in blue [3,4]; (b) Spatial arrangement of FAD cofactor and tryptophan residues, selected edge-to-edge distances in $\AA$; (c) RMSD from a $160 \mathrm{~ns}$ trajectory of dCRY (blue) and CTT (orange, residues 515-537); (d) Zoom of CTT rmsd for the considered time interval in QM/MM simulations. (e) Excitation energies of the local excited $\pi \pi^{*}$ state of FAD (blue) and charge transfer states $\mathrm{ISO}^{-} \mathrm{W} 420^{+}$(red), $\mathrm{ISO}^{-} \mathrm{ADE}^{+}$(grey) and $\mathrm{ISO}^{-} \mathrm{W} 314^{+}$(green). Excitation energies were evaluated on QM/MM TD-DFT(BNL/cc-pvdz) level every $125 \mathrm{ps}$ (dashed) and are shown together with the moving average (solid).

\section{MACGIC-QUAPI simulations of electron transfer dynamics}

Microscopically derived Hamiltonians were obtained by evaluating QM/MM excitations energies along a $\sim 50$ ns segment of a 160 ns dCRY MD trajectory (Fig. 1c-e), inherently accounting for enzyme thermal fluctuations of charge separated states. Additional trajectories conducted in respective charge separated states (e.g. $\mathrm{ISO}^{-} \mathrm{W} 420^{+}, \mathrm{ISO}^{-} \mathrm{W} 314^{+}$ etc.) show convergence to Gaussian statistics and allow for the reliable construction of free energy differences $\Delta \mathrm{G}$ and reorganization energies of participating charge separated states within the enzyme environment (Fig. 2a-b) [5]. Non-Markovian real-time evolution of charge separation dynamics is simulated with the recently introduced MACGIC-QUAPI method [6] that relies on a non-linear intermediate representation of the influence functional and provides convergence to exact HEOM benchmark results for arbitrary system-bath coupling strength.

We find that due to the comparable energetics of charge separated states an assignment to a unique charge separation pathway, either the TRP-triad pathway $\left(\mathrm{ISO}^{-} \mathrm{W} 420^{+}-\mathrm{ISO}^{-} \mathrm{W} 397^{+}\right.$) or the alternative pathway towards the CTT $\left(\mathrm{ISO}^{-} \mathrm{ADE}^{+}\right.$$\mathrm{ISO}^{-} \mathrm{W} 314^{+}$), is precluded. The wild-type enzyme charge separation dynamics confirms the picture that both pathways contribute to the deactivation of the initially excited $\pi \pi^{*}$ state of ISO (Fig. 2c, top) which is depopulated on the tens-of-picosecond timescale, leading to a parallel population of $\mathrm{ISO}^{-} \mathrm{ADE}^{+}$and $\mathrm{ISO}^{-} \mathrm{W} 420^{+}$charge separated states, followed by depopulation into $\mathrm{ISO}^{-} \mathrm{W} 314^{+}$and $\mathrm{ISO}^{-} \mathrm{W} 397^{+}$states.

The effect of enzyme environment is investigated by in silico mutations of selected amino acid residues. We find that a small set of charged residues strongly affects the energetics of charge separated states, which in part have destabilizing impact on the TRPtriad pathway and stabilizing effect on the pathway involving TRP314. Accordingly, the dynamics of in silico mutated dCRY (Fig 2c, bottom) is significantly altered, showing 
ultrafast depopulation of the ISO $\pi \pi^{*}$ state on the 1-2 ps timescale with population of the $\mathrm{ISO}^{-} \mathrm{ADE}^{+}-\mathrm{ISO}^{-} \mathrm{W} 314^{+}$pathway only. Our results highlight the importance of CTT directional charge separation events in dCRY in addition to the conventional Trp-triad direction.

(a)

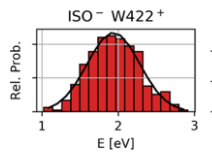

(b)

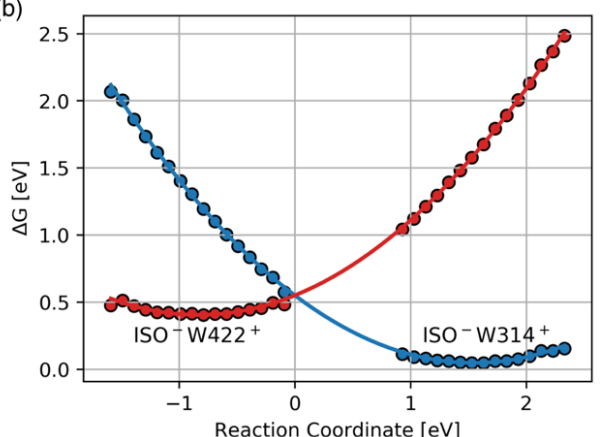

(c)
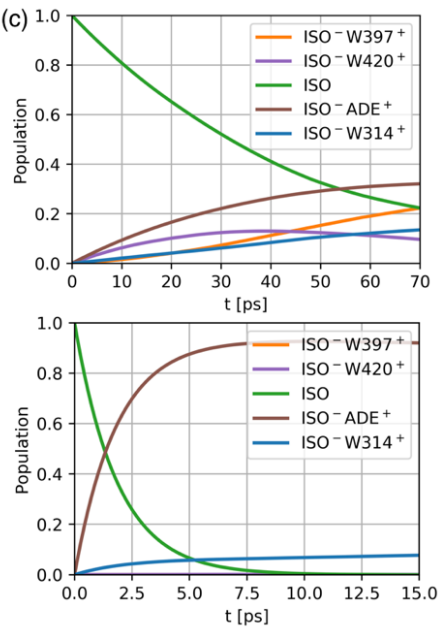

Fig. 2. (a) Representative energy histogram of $\mathrm{ISO}^{-} \mathrm{W} 422^{+}$and $\mathrm{ISO}^{-} \mathrm{W} 314^{+}$charge separated states and respective energy difference distribution employed for calculation of free energy surfaces shown in (b) [5]; presented data in (a) for a trajectory evolving in the $\mathrm{ISO}^{-} \mathrm{W}_{422^{+}}$state. (c) Population dynamics of wild-type dCRY (top) and in silico mutated dCRY (bottom), evaluated with the MGACIC-QUAPI method [6] (top: 2048 time steps memory interval $=130.3 \mathrm{fs}, 64$ effective mask elements; bottom: 128 time steps memory interval, 32 effective mask elements).

B.P.F. gratefully acknowledges support through the DFG within the Emmy Noether Programme (Grant No. FI 2034/1-1).

\section{References}

1. N. Ozturk, C. P. Selby, D. Zhong, A. Sancar, J. Biol. Chem. 289, 4634 (2014).

2. A. T. Vaidya, D. Top, C. C. Manahan, J. M. Tokuda, S. Zhang, L. Pollack, M. W. Young, B. R. Crane, Proc. Nat. Acad. Sci. 110, 20455 (2013).

3. C. Levy, B. D. Zoltowski, A. R. Jones, A. T. Vaidya, D. Top, J. Widom, M. W. Young, N. S. Scrutton, B. R. Crane, and D. Leys, Nature 495, E3 (2011).

4. A. Czarna, A. Berndt, H. Raj Singh, A. Grudziecki, A. G. Ladurner, G. Timinszky, A. Kramer, E. Wolf., Cell 153, 1394 (2013).

5. W. W. Parson, Z. T. Chu, A. Warshel, Biophys. J. 74, 182 (1998).

6. M. Richter, B. P. Fingerhut, J. Chem. Phys. 146, 214101 (2017). 